\title{
Separation of Mycobacterium abscessus into subspecies or genotype level by direct application of peptide nucleic acid multi-probe- real-time PCR method into sputa samples
}

Kijeong Kim², Seok-Hyun Hong ${ }^{1}$, Byoung-Jun Kim, Bo-Ram Kim', So-Young Lee', Ga-Na Kim', Tae Sun Shim³, Yoon-Hoh Kook ${ }^{1}$ and Bum-Joon Kim ${ }^{1 *}$

\begin{abstract}
Background: Recently, we introduced a novel peptide nucleic acid (PNA) multi-probe real time PCR method targeting the hsp65 gene (hsp65 PNA RT-PCR) to distinguish Mycobacterium abscessus groups.

Methods: Here, we evaluated the usefulness of the hsp65 PNA RT-PCR for the direct identification of the $M$. abscessus group at the subspecies and genotype levels from sputa samples. The method was applied to total sputa DNA from 60 different patients who were identified as having mycobacterial infections via rpoB PCR restriction analysis of the same cultures.

Results: The hsp65 PNA RT-PCR method had higher sensitivity than the multi-probe real-time PCR assay targeting hsp65 (HMPRT-PCR) for the detection of M. abscessus from sputum [96.7 \% (29/30 samples) vs. 70 \% (21/30 samples); $100 \%$ specificity].
\end{abstract}

Conclusions: These results suggest that the PNA-based method is feasible for the detection of $M$. abscessus members not only from cultures but also directly from sputa.

Keywords: Mycobacterium abscessus, Mycobacterium massiliense, Peptide nucleic acid (PNA), Real time PCR, hsp65, Genotype

\section{Background}

The Mycobacterium abscessus complex represents a group of rapidly growing mycobacteria (RGM) that account for approximately $65-80 \%$ of RGM pulmonary infections [1]. In Korea, the incidence of $M$. abscessus lung diseases has been increasing; these infections account for $70-80 \%$ of RGM-induced lung diseases [2-5].

The taxonomic status of the M. abscessus group remains undetermined. Recent advances in taxonomic approaches revealed that the $M$. abscessus group could be further divided into three closely related taxa [i.e., $M$. abscessus subsp. abscessus (hereafter referred to as $M$.

\footnotetext{
* Correspondence: kbumjoon@snu.ac.kr

'Department of Biomedical Sciences, Microbiology and Immunology, Cancer Research Institute, and Liver Research Institute, Seoul National University College of Medicine, 28 Yongon-dong, Chongno-gu, Seoul 110-799, Republic of Korea
} Full list of author information is available at the end of the article abscessus), M. abscessus subsp. massiliense (hereafter referred to as $M$. massiliense) and $M$. abscessus subsp. bolletii (hereafter referred to as M. bolletii)] [6-8]. Recently, it was reported that M. massiliense could be further subdivided into two genotypes (hereafter referred to as Type I and Type II) based on hsp 65 sequence analysis. All of the strains belonging to Type II are distinct in Korean patients due to a rough colony morphotype without any exceptions [9]. A recent complete genome study revealed that the rough colony phenotype of the Type II strains may be due to a large deletion event in the glycopeptidolipid (GPL)-related genes [10, 11]. Furthermore, a recent report based on pulsed-field gel electrophoresis (PFGE) and multi-locus sequence typing (MLST) also revealed taxonomic separation between $M$. massiliense Type II-1 and Type II-2 [12].

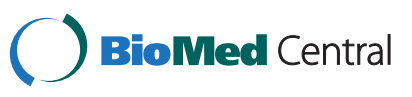

(c) 2015 Kim et al. Open Access This article is distributed under the terms of the Creative Commons Attribution 4.0 International License (http://creativecommons.org/licenses/by/4.0/), which permits unrestricted use, distribution, and reproduction in any medium, provided you give appropriate credit to the original author(s) and the source, provide a link to the Creative Commons license, and indicate if changes were made. The Creative Commons Public Domain Dedication waiver (http://creativecommons.org/publicdomain/zero/1.0/) applies to the data made available in this article, unless otherwise stated. 
M. massiliense has been increasingly recognized as an emerging pathogen that causes postsurgical wound infection outbreaks [13]. Recently, it was identified as the causative agent of respiratory outbreaks in two cystic fibrosis centers and showed evidence of patient-to-patient transmission $[14,15]$. Infections with the $M$. abscessus group are difficult to treat, because these mycobacteria exhibit intrinsic resistance to various antibiotics. Susceptibility to clarithromycin (CLA) varies among members of the $M$. abscessus group; for example, while the majority of $M$. abscessus and $M$. bolletii exhibit resistance to CLA, M. massiliense is susceptible. Resistance is characterized by the presence of the erm(41) gene that encodes the erythromycin ribosomal methylases (ERM) [16].

Despite their close genetic relatedness, disparities in pathogenic potential, transmission mode, and antibiotic susceptibility were found among members of the $M$. abscessus group. Therefore, accurate identification of members of the M. abscessus group is important for patient treatment and epidemiological purposes [17].

Peptide nucleic acids (PNAs) are artificially synthesized DNA analogues with an uncharged peptide backbone. PNAs have more favorable hybridization properties and chemical, thermal, and biological stability due to their uncharged nature and peptide bond-linked backbone [18]. Due to these favorable characteristics, PNA has been widely applied as a diagnostic tool in molecular biology [19]. A PNA probe-based real-time PCR assay has been developed for mycobacteria diagnosis, particularly for the simultaneous separation of $M$. tuberculosis and NTM in clinical specimens $[20,21]$. Recently, we introduced a novel peptide nucleic acid (PNA) multi-probe real- time PCR method that targeted the $h s p 65$ gene ( $h s p 65$ PNA RT-PCR) to distinguish between the four types within the $M$. abscessus groups $[M$. abscessus and the $3 M$. massiliense types (Type I, Type II-1 and Type II-2)] using 3 PNA probes. For this evaluation, we applied 27 reference strains and 228 clinical isolates belonging to the M. abscessus groups. With the exception of one clinical isolate, most of the samples (227/ 228 isolates, $99.6 \%$ sensitivity) were clearly separated at the subspecies or genotype levels, thereby demonstrating the technique's feasibility for the detection of $M$. abscessus at the mycobacterial culture level. To the best of our knowledge, this was the first report to use a PNA- based multiprobe approach for bacterial diagnosis [22].

The aim of this study was to evaluate the usefulness of the hsp65 PNA RT-PCR in directly identifying the $M$. abscessus group at the subspecies or genotype levels from sputum samples. We applied the technique to sputum DNA samples from 60 different patients who were previously diagnosed with mycobacterial infections via rpoB PCR restriction analysis of the same cultures (30 samples were culture positive for $M$. abscessus complex strains, and 30 samples were culture positive for other mycobacteria). These results were compared with the results from the multi-probe real-time PCR assay targeting hsp65 (HMPRT-PCR) that was previously developed by our laboratory [23].

\section{Methods}

\section{Mycobacterial strains and sputum samples}

Six mycobacteria reference strains including 5 strains belonging to the $M$. abscessus group (M. abscessus ATCC $19977^{\mathrm{T}}$, M. bolletii CIP $108541^{\mathrm{T}}$, M. massiliense Type I KCTC $19086^{\mathrm{T}}$, M. massiliense Type II-1 SNUMC 50594 and $M$. massiliense Type II-2 SNUMC 53618) and $M$. tuberculosis H37Rv ATCC $27294^{\mathrm{T}}$, and 60 sputum specimens suspected of harboring mycobacterial infection were used in this study. Four of the 6 mycobacterial reference strains were provided by the Korean Institute of Tuberculosis (KIT). M. massiliense Type II-1 SNUMC 50594 and $M$. massiliense Type II-2 SNUMC 53618 were provided by the Seoul National University College of Medicine (SNUMC). Sixty sputum samples from different patients with positive AFB smears detected between April 01, 2008, and July 31, 2008, at AMC, Seoul, Republic of Korea, were included in this study. Identification of mycobacterial infections in all sputum samples (30 samples were culture positive for M. abscessus complex strains and 30 samples were culture positive for other mycobacteria) was previously determined by rpoB PRA analysis of the same cultures. The sputa were digested, decontaminated, and concentrated as recommended by the WHO [24]. The processed sediment was stained using the Ziehl-Neelsen method. The results of the AFB smears were graded according to the recommendations of the American Thoracic Society and the Center for Disease Control and Prevention [25]. Sputa with trace AFB smear results (1-2 bacilli in 300 fields) were also included in this study. The protocol for this study including the documentation for waiver of informed consent was approved by the institutional review board of Seoul National University Hospital (C-1503-058-655) and Asan Medical Center (AMC IRB 2007-0331).

\section{DNA extraction}

Chromosomal DNA was extracted from the sputum samples using the bead beater-phenol extraction method as previously described [26].

\section{hsp65 PNA RT-PCR}

For the detection of $M$. abscessus from sputum samples, the hsp65 PNA RT-PCR method was applied to $60 \mathrm{spu}-$ tum DNA samples, as previously described [22]. Briefly, a total of three reporter dyes were used for the specific simultaneous detection of the four types of the M. abscessus group (M. abscessus, Type I, Type II-1, and Type II-2) in a single reaction.: FAM for the detection of $M$. abscessus 
and M. massiliense at the species level, Hex for the discrimination of $M$. massiliense Type I and Type II, and Texas Red for the discrimination of $M$. massiliense Type II-1 and Type II-2. The sequences of the probes and primers are provided in Table 1 . The probes were purchased from Panagene (Daejeon, Korea) and the primers from Macrogen (Seoul, Korea). A LightCycler (version 96; Roche Life Science, Mannheim, Germany) system was used for the real-time PCR, and three channels were used for the experiment. The optimal reaction mixture was established for the sensitive and specific detection of target sequences. A $10 \mu \mathrm{l}$ reaction mixture was prepared for each sample as follows: $1 \mu \mathrm{l}$ PCR reaction buffer for FastStart Taq (Roche), $2 \mathrm{mM} \mathrm{MgCl} 2,2 \mu \mathrm{l}$ GC-RICH solution (Roche), $0.2 \mathrm{mM}$ deoxynucleoside triphosphate mixture (Roche), $1 \mu \mathrm{M}$ forward primer (Abs/Mas-F, 5'-CCGA GACGCTGCTGAAGAG-3'), $0.3 \mu \mathrm{M}$ reverse primer (Abs/Mas-R, 5' -GACGTCCTCGGCGATGAT-3'), $0.4 \mu \mathrm{M}$ PNA FAM probe (Mas/Abs, 5'-Dabcyl-CCTCGTTAC CAACCT-O-K-FAM-3'), $0.15 \mu$ M PNA Hex probe (MasT2, 5'-Dabcyl-GGAGATTCCGGCC-O-K-Hex-3'), $0.5 \mu \mathrm{M}$ PNA Texas Red probe (Mas-T2-1), 0.4 U FastStart Taq (Roche), $0.1 \mathrm{mg} / \mathrm{ml}$ bovine serum albumin (New England Biolabs), $2 \mu \mathrm{l}$ template DNA, and distilled water (Roche). The cycling conditions were $300 \mathrm{~s}$ at $95^{\circ} \mathrm{C}$ and 45 cycles of $20 \mathrm{~s}$ at $95^{\circ} \mathrm{C}, 15 \mathrm{~s}$ at $62{ }^{\circ} \mathrm{C}$ (single acquisition of fluorescence signals), and $40 \mathrm{~s}$ at $74{ }^{\circ} \mathrm{C}$. Melting curve analysis was performed using the following cycles: $120 \mathrm{~s}$ at $95{ }^{\circ} \mathrm{C}$ and $180 \mathrm{~s}$ at $37{ }^{\circ} \mathrm{C}$ with a ramping speed of $1.1^{\circ} \mathrm{C} / \mathrm{s}$. Then, the temperature was increased from $37{ }^{\circ} \mathrm{C}$ to $80{ }^{\circ} \mathrm{C}$ at a temperature transition rate of $0.07{ }^{\circ} \mathrm{C} / \mathrm{s}$ during which time the fluorescence signal was continuously acquired. Duplicate experiments were performed to determine the melting temperatures of the probes designed for the target $M$. abscessus group by real-time PCR, and DNA from a total of 60 sputum samples (Additional file 1) was subsequently tested for species identification by $T_{m}$ analysis.

\section{HMPRT-PCR}

The HMPRT-PCR was applied to the same 60 sputum DNA samples as previously described for comparison with the hsp65 PNA RT-PCR [23]. Briefly, a total of 4 channels $(\mathrm{CH}$.) were used for probes specific for 7 mycobacteria species: CH. 610 for the detection of M. tuberculosis, CH. 640 for the detection of $M$. kansasii, $M$. intracellulare, and $M$. fortuitum-peregrinum complex, $\mathrm{CH}$. 670 for the detection of $M$. avium, and $\mathrm{CH} .705$ for the detection of M. abscessus and M. massiliense. A LightCycler 2.0 system was used for the real- time PCR. The LC Faststart DNA Master HP kit (Roche Diagnostics) was used for the preparation of the mastermix according to the kit protocol. A $10 \mu \mathrm{l}$ reaction mixture was prepared for each sample as follows: $1 \mu \mathrm{l}$ Taq buffer (containing dNTP mix and $10 \mathrm{mM} \mathrm{MgCl}_{2}$ ), an additional $2 \mathrm{mM} \mathrm{MgCl} 2,0.4 \mu \mathrm{M}$ NTM-specific forward primer (HSP-NTM-F, 5'-CCGYTGCTGGAGAAGGTCA TYCAG-3'), $0.3 \mu \mathrm{M}$ TBC-specific forward primer (HSPTBC-F, 5' -CGCTGCTCGAGAAGGTCATCGGA-3'), $1 \mu \mathrm{M}$ mycobacteria-specific reverse primer (HSP-MYC-R, 5' CGATGATGGTGGTCTCGTCCTTGGT-3'), $0.2 \mu \mathrm{M}$ HybProbes, template $(2 \mu \mathrm{l}$ of culture-extracted DNA or $4 \mu \mathrm{l}$ of sputum-extracted DNA with $0.1 \mathrm{mg} / \mathrm{ml}$ BSA (NEB)) and sterile distilled water. The cycling conditions were as follows: $10 \mathrm{~min}$ at $95^{\circ} \mathrm{C}$ and 45 cycles of $10 \mathrm{~s}$ at $95^{\circ} \mathrm{C}, 20 \mathrm{~s}$ at $65^{\circ} \mathrm{C}$ (single acquisition of fluorescence signals) and $20 \mathrm{~s}$ at $72{ }^{\circ} \mathrm{C}$. The cycling was followed by melting curve analysis: $10 \mathrm{~s}$ at $95^{\circ} \mathrm{C}$ and $30 \mathrm{~s}$ at $57^{\circ} \mathrm{C}$. The temperature was increased from $57{ }^{\circ} \mathrm{C}$ to $90{ }^{\circ} \mathrm{C}$ with a temperature transition rate of $0.1{ }^{\circ} \mathrm{C} / \mathrm{s}$ and the continuous acquisition of the fluorescence signal.

\section{Results}

Application of $h s p 65$ PNA RT-PCR to 30 sputum samples previously demonstrated to be infected by the M. abscessus group

To evaluate the usefulness of hsp65 PNA RT-PCR for the direct detection of $M$. abscessus group infection from sputa DNA, we tested a total of 30 sputum DNA samples from different patients previously diagnosed with infection by the $M$. abscessus group via rpoB PCR restriction analysis of the same cultures. The hsp65 PNA RT-PCR method enabled the identification of 29 out of 30 sputum samples (96.7 \%) into 18 M. abscessus and 11

Table 1 Primers and PNA probes used for identification of Mycobacterium abscessus group in the present study

\begin{tabular}{|c|c|c|c|}
\hline$\underline{\text { Primer/Probe }}$ & Sequence $\left(5^{\prime} \rightarrow 3^{\prime}\right)$ & $T_{m}\left({ }^{\circ} \mathrm{C}\right)^{a}$ & Potential target organisms \\
\hline \multicolumn{4}{|l|}{ Primer } \\
\hline Abs/Mas-F & CCGAGACGCTGCTGAAGAG & 66.2 & M. abscessus, M. massiliense \\
\hline Abs/Mas-R & GACGTCCTCGGCGATGAT & 65.5 & M. abscessus, M. massiliense \\
\hline \multicolumn{4}{|l|}{ PNA Probe } \\
\hline Mas/Abs & Dabcyl-CCTCGTTACCAACCT-O-K-FAM & 66.8 & M. massiliense, M. abscessus \\
\hline Mas-T2 & Dabcyl-GGAGATTCCGGCC-O-K-Hex & 69.5 & M. massiliense Type 2/Type 1 \\
\hline Mas-T2-1 & Dabcyl-CTGAATTACCTTCTC-O-K-Texas Red & 62.0 & M. massiliense Type 2-1/Type 2-2 \\
\hline
\end{tabular}

${ }^{a}$ Primer $T_{m}$ was calculated by using Oligo software $\mathrm{V} 6.50$ and probe $T_{m}$ was calculated by http://pnabio.com/support/PNA_Tool.htm; $T_{m}$, melting temperature ${ }^{b} \mathrm{~K}$, lysine; $\mathrm{O}$, O linker 
M. massiliense strains. The assay was also able to differentiate all $11 \mathrm{M}$. massiliense strains into three genotypes: Type I ( 5 sputa), Type II-1 (4 sputa) and Type II-2 (2 sputa) (Fig. 1a-c, Table 2 and Additional file 1).

To verify the consistency of our hsp 65 PNA RT-PCR method in a clinical setting, we analyzed the range of $T_{m} \mathrm{~s}$ of the clinical samples at the intra-subspecies or intra-genotypic level in each channel. In the FAM channel, differences in $T_{m} \mathrm{~s}$ among the $18 \mathrm{M}$. abscessus sputum samples and $11 \mathrm{M}$. massiliense strains were $2.9^{\circ} \mathrm{C}$ $\left(49.0-52.3{ }^{\circ} \mathrm{C}\right)$ and $0.7{ }^{\circ} \mathrm{C}\left(61.4-62.1{ }^{\circ} \mathrm{C}\right)$, respectively, which was less than the inter-subspecies $T_{m} \mathrm{~s}$ differences between $M$. abscessus and M. massiliense (11.5-11.9 $\left.{ }^{\circ} \mathrm{C}\right)$. In the Hex channel, differences in $T_{m} \mathrm{~s}$ among the $4 M$. massiliense Type I and $6 \mathrm{M}$. massiliense Type II sputum samples were $1.0{ }^{\circ} \mathrm{C}\left(60.0-61.0{ }^{\circ} \mathrm{C}\right)$ and $1.1{ }^{\circ} \mathrm{C}(68.5-$ $69.6{ }^{\circ} \mathrm{C}$ ), respectively, which was less than the intersubspecies $T_{m} \mathrm{~s}$ difference between $M$. massiliense Type I and Type II $\left(8.8-9.1{ }^{\circ} \mathrm{C}\right)$. In the Texas Red channel, the differences in $T_{m} \mathrm{~s}$ among the $4 M$. massiliense Type II-1 and $2 M$. massiliense Type II-2 sputum samples were $1.9^{\circ} \mathrm{C}\left(51.5-53.4{ }^{\circ} \mathrm{C}\right)$ and $0.3{ }^{\circ} \mathrm{C}\left(46.4-46.7{ }^{\circ} \mathrm{C}\right)$, respectively, which was less than the inter-genotypic $T_{m} \mathrm{~s}$ difference between $M$. massiliense Type II-1 and Type II-2 $\left(4.8-7{ }^{\circ} \mathrm{C}\right)$ (Table 3). These results demonstrate the suitability of the $h s p 65$ PNA RT-PCR for the separation of the $M$. abscessus group into subspecies or genotypes in clinical settings.

\section{Application of $h s p 65$ PNA RT-PCR to 30 sputum samples previously demonstrated to be infected by mycobacteria other than the $M$. abscessus group}

To validate the specificity of the $h s p 65$ PNA RT-PCR method in detecting $M$. abscessus group infections from sputum samples, we applied the technique to 30 sputum samples from different patients previously identified as infected with mycobacteria other than the $M$. abscessus group (10 patients with $M$. tuberculosis, 9 with $M$. avium, 7 with $M$. intracellulare, 1 with $M$. celatum, 1 with $M$. fortuitum, 1 with $M$. kansasii and 1 with $M$. szulgai) via rpoB PCR restriction analysis of the same cultures. None of the 30 sputum DNA samples produced a detectable $T_{m}$

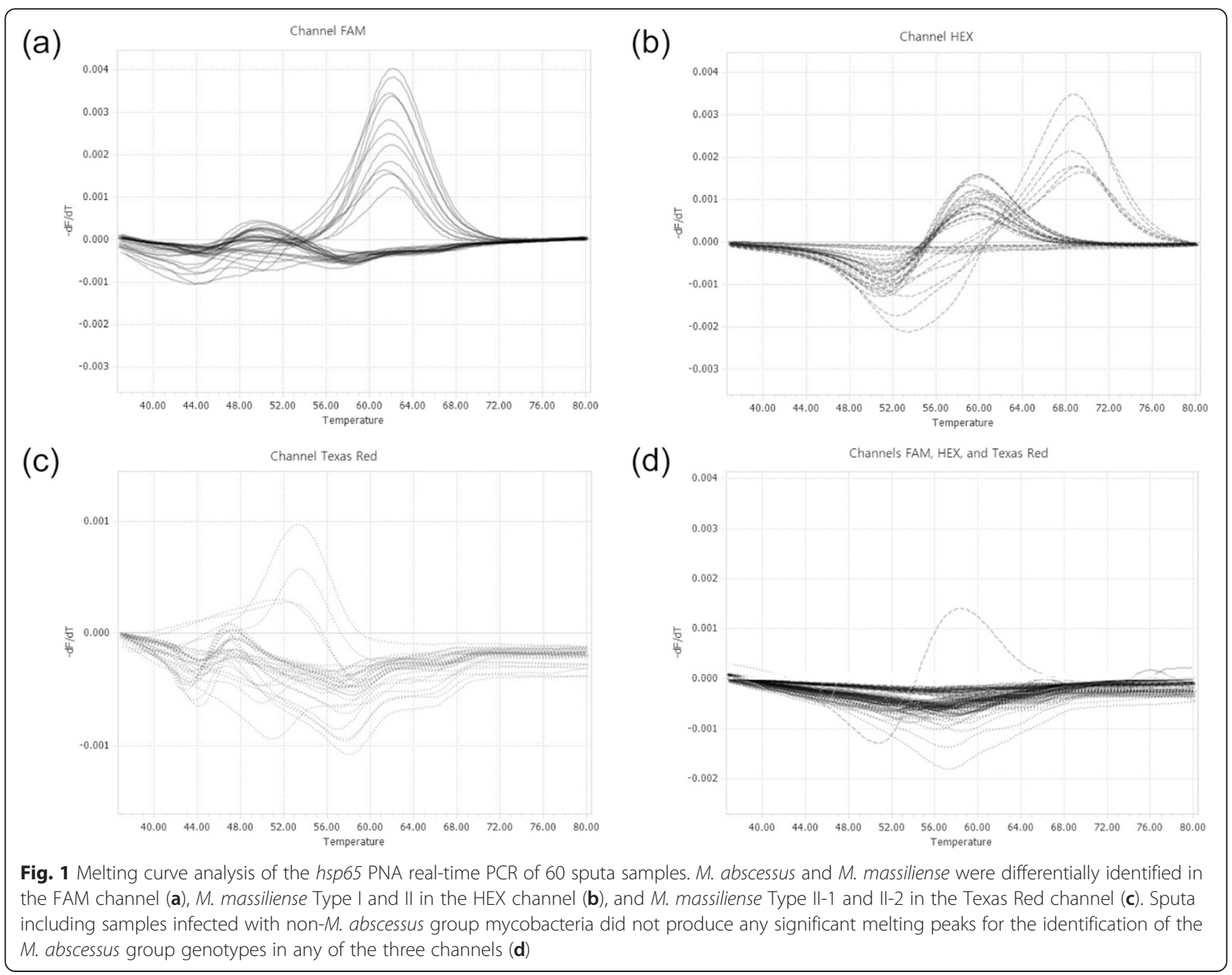


Table 2 Comparison of identification results between sputum hsp65 PNA real-time PCR and sputum HMPRT-PCR based on same culture-based rpoB PRA

\begin{tabular}{|c|c|c|c|}
\hline & rpoB PRA & HMPRT-PCR & $\begin{array}{l}\text { hsp65 PNA } \\
\text { real-time PCR }\end{array}$ \\
\hline \multicolumn{4}{|l|}{ Sensitivity } \\
\hline M. abscessus & 30 & 14 & 18 \\
\hline M. massiliense & NA & 7 & 11 \\
\hline M. massiliense Type I & NA & NA & 5 \\
\hline M. massiliense Type II-1 & NA & NA & 4 \\
\hline M. massiliense Type II-2 & NA & NA & 2 \\
\hline Total No. of identification & & $21(70 \%)$ & $29(97 \%)$ \\
\hline No. of misidentification & & 0 & 0 \\
\hline \multicolumn{4}{|l|}{ Specificity } \\
\hline Non-M. abscessus group & 30 & 28 & 0 \\
\hline $\begin{array}{l}\text { M. abscessus group } \\
\text { identified }\end{array}$ & & 0 (100\%) & 0 (100\%) \\
\hline No. of misidentification & & 0 & NA \\
\hline
\end{tabular}

$\mathrm{NA}$, not available

Table 3 Measurement of melting temperatures of $M$. abscessus group from sputa samples by hsp65 PNA real-time PCR

\begin{tabular}{|c|c|c|c|}
\hline \multirow[b]{2}{*}{ Species } & \multicolumn{3}{|l|}{ Channels } \\
\hline & FAM & Hex & Texas Red \\
\hline \multicolumn{4}{|l|}{ M. abscessus } \\
\hline No. of identification & 18 & 18 & 18 \\
\hline Average $T_{m}\left({ }^{\circ} \mathrm{C}\right)^{b}$ & $50.0 \pm 0.85$ & $59.7 \pm 0.37$ & $47.1 \pm 0.45$ \\
\hline Range of $T_{m}\left({ }^{\circ} \mathrm{C}\right)$ & $49.0-52.3$ & $59.0-60.5$ & $46.3-47.7$ \\
\hline No. of failed detection & 0 & 0 & 3 \\
\hline \multicolumn{4}{|l|}{ M. massiliense Type I } \\
\hline No. of identification & 5 & 5 & 5 \\
\hline Average $T_{m}\left({ }^{\circ} \mathrm{C}\right)$ & $61.8 \pm 0.37$ & $60.6 \pm 0.56$ & $46.9 \pm 0.28$ \\
\hline Range of $T_{m}\left({ }^{\circ} \mathrm{C}\right)$ & $61.4-62.1$ & $59.9-61.0$ & $46.5-47.2$ \\
\hline No. of failed detection & 0 & 0 & 0 \\
\hline \multicolumn{4}{|l|}{ M. massiliense Type II-1 } \\
\hline No. of identification & 4 & 4 & 4 \\
\hline Average $T_{m}\left({ }^{\circ} \mathrm{C}\right)$ & $61.9 \pm 0.08$ & $69.1 \pm 0.49$ & $52.7 \pm 0.89$ \\
\hline Range of $T_{m}\left({ }^{\circ} \mathrm{C}\right)$ & $62.0-63.3$ & $68.4-71.3$ & $51.5-53.4$ \\
\hline No. of failed detection & 0 & 0 & 0 \\
\hline \multicolumn{4}{|l|}{ M. massiliense Type II-2 } \\
\hline No. of identification & 2 & 2 & 2 \\
\hline Average $T_{m}\left({ }^{\circ} \mathrm{C}\right)$ & $62.0 \pm 0.07$ & $69.0 \pm 0.42$ & $46.6 \pm 0.21$ \\
\hline Range of $T_{m}\left({ }^{\circ} \mathrm{C}\right)$ & $61.9-62.0$ & $68.7-69.3$ & $46.4-46.7$ \\
\hline No. of failed detection & 0 & 0 & 0 \\
\hline
\end{tabular}

in the FAM channel that was specific for M. abscessus group species detection, thereby validating the high specificity of the hsp65 PNA RT-PCR method for the detection of the $M$. abscessus group from sputum samples. Only one sputum sample (M. fortuitum) formed an insignificant $T_{m}$ in the Hex channel (Fig. 1d).

\section{Comparison of $h s p 65$ PNA RT-PCR versus HMPRT-PCR for the direct detection of the $M$. abscessus group from sputum samples}

Previously, we introduced a multiprobe real-time PCR assay targeting hsp65 (HMPRT-PCR) to detect and identify pathogenic mycobacteria directly from sputum specimens. In this study, we compared the 2 methods ( $h s p 65$ PNA RT-PCR and HMPRT-PCR) for the direct detection of the $M$. abscessus group from sputum samples. When the 2 methods were applied to the same 30 sputum DNA samples, the results showed that the hsp65 PNA RT-PCR had higher sensitivity compared to the HMPRT-PCR in detecting $M$. abscessus from sputum [96.7 \% (29/30 samples) vs. $70 \%$ (21/30samples)] (Table 2). A discrepancy between the two methods was found in nine samples. One sputum sample (S1380) identified as M. massiliense Type II-1 infection by $h s p 65$ PNA RT-PCR was identified as $M$. avium infection by HMPRT-PCR; its culture identification was confirmed to be a coinfection of $M$. abscessus and $M$. avium. HMPRT-PCR failed to detect $M$. abscessus in this coinfection. Another sample (S1479) identified as M. massiliense Type II-2 infection by hsp65 PNA RT-PCR was identified as a coinfection of $M$. massiliense and M. avium HMPRT-PCR; its culture identification was M. abscessus infection. HMPRT-PCR could not detect 5 samples identified as $M$. abscessus infections by hsp65 PNA RT-PCR or two samples identified as $M$. massiliense Type I and Type II-1 infections by hsp65 PNA RT-PCR (Additional file 1).

\section{Discussion}

Recently, we reported that phylogenetic analysis based on the sequence of the 604-bp hsp65 gene enabled taxonomic separation among members of the $M$. abscessus groups at the genotype and subspecies levels despite representing a single target gene [9]. We also reported that the separation of members of the M. abscessus group based on pulsed-field gel electrophoresis (PFGE) and multi-locus sequence typing (MLST) was nearly in agreement with the results obtained using sequencebased phylogenetic analysis of the 604-bp hsp65 gene [12], which resulted in the phylogenetic separation of four members of the $M$. abscessus groups [M. abscessus and the three types of $M$. massiliense (I, II-1 and II-2)].

SNPs have been widely used for the diagnostic targeting of pathogens $[27,28]$ and human diseases [29]. For use in bacterial identification, an SNP should show not only intra-species or genotypic conservation but also 
inter-species or genotypic variation. Notably, there are several SNPs in the 604-bp $h s p 65$ sequence that are potential targets of diagnostic methods for the separation of members of $M$. abscessus. In a previous study, we developed an $h s p 65$ PNA RT-PCR targeting three signature SNPs in the 604-bp hsp65 gene that enabled the separation of the four types of M. abscessus (M. abscessus and three types of $M$. massiliense) at the cultured isolate level. The intra-genotypic conservation and intergenotypic variation for each SNP of members of the $M$. abscessus group were confirmed by successful separation in 227 out of 228 clinical isolates (99.6\%) [22]. In this study, the application of the hsp65 PNA RT-PCR method to sputum samples demonstrated that this method could directly identify members of the $M$. abscessus group at the subspecies or genotypic level from sputum samples with $96.7 \%$ sensitivity (29/30 samples) and $100 \%$ specificity (Table 2), thereby demonstrating its feasibility in identifying the $M$. abscessus group not only from cultured isolates but also directly from sputum samples.

PNA-based molecular beacons were reported to be superior to conventional molecular probes due to their faster hybridization kinetics, high signal to background ratio and improved specificity [30]. Indeed, PNAs have been successfully applied for the highly sensitive detection of anthrax DNA and HIV RNA [31, 32]. PNA-based molecular beacons were reported to be advantageous for genotyping short sequences when high sequence specificity was required. The typing of an SNP with DNA probes such as the TaqMan or LightCycler probes (which are usually at least 23 nucleotides in length) can be problematic due to the limited discriminating power of long DNA probes. PNA molecular probes are significantly shorter than the TaqMan or LightCycler probes, making probe design and genotype discrimination easier [33]. Despite the short sequence length (13-15 bp) of the probes, our hsp65 PNA RT-PCR could be successfully applied to the separation of members of the $M$. abscessus group from sputum samples (Table 1) as well as clinical isolates [22]. The presence of a mismatch in a PNA/DNA duplex is reported to be more destabilizing than a mismatch in a DNA/DNA duplex, suggesting that the use of PNA probes may lead to more pronounced difference in the average $T_{m}$ in SNP detection compared to the use of DNA probes [34]. In this study, our PNA probes showed relatively large differences $\left(4.8{ }^{\circ} \mathrm{C}\right.$ to $11.9^{\circ} \mathrm{C}$ ) in $T_{m} \mathrm{~s}$ in SNP detection despite one nucleotide mismatch. Thus, our probes provided improved discriminative power for the separation of members of the $M$. abscessus group compared to DNA probes, which are always higher compared to the probes used for intrasubspecies or intra-genotype analyses. This result demonstrates the feasibility and reproducibility of our $h s p 65$
PNA RT-PCR method in a clinical setting. The comparison of hsp65 PNA RT-PCR versus HMPRT-PCR in this study demonstrated the higher sensitivity of the former compared to the latter [96.7 \% (29/30 samples) vs. $70 \%$ (21/30 samples)] in detecting M. abscessus from sputum samples. These findings strongly support the hypothesis that PNA-probe based technology may be superior to technologies based on other probes (i.e., FRET-based dual probes for the direct detection of mycobacteria from sputum samples).

Notably, the $T_{m} \mathrm{~s}$ measured from sputum samples showed small decreasing shifts $\left(1-2.7^{\circ} \mathrm{C}\right)$ compared to our previously reported $T_{m} \mathrm{~s}$ of clinical isolates of the $M$. abscessus genotypes [22] without compromising the discrimination of the genotypes. This outcome may be due to the presence of PCR-inhibitory substances in the DNA extracts from the sputum specimens because inhibitory substances can cause $T_{m}$ shifts $[35,36]$.

\section{Conclusions}

In conclusion, our data suggest that the hsp65 PNA RTPCR method developed in the previous study may represent a promising approach for identifying the subspecies or genotypes of the $M$. abscessus group directly from sputum samples.

\section{Additional file}

Additional file 1: Identification of $M$. abscessus and $M$. massiliense genotypes from DNA extracts of sputa samples using hsp65 PNA real-time PCR. (DOCX $19 \mathrm{~kb}$ )

\section{Abbreviations}

AFB: Acid-fast bacillus; CLA: Clarithromycin; ERM: Erythromycin ribosomal methylase; GPL: Glycopeptidolipid; KIT: Korean Institute of Tuberculosis; MLST: Multi-locus sequence typing; NTM: Nontuberculous mycobacteria; PFGE: Pulsed-field gel electrophoresis; PNA: Peptide nucleic acid; PRA: PCRrestriction enzyme analysis; RGM: Rapidly growing mycobacteria; SNP: Single nucleotide polymorphism; SNUMC: Seoul National University College of Medicine.

\section{Competing interests}

The authors declare that they have no competing interests.

\section{Authors' contributions}

$\mathrm{KK}$ and $\mathrm{SHH}$ performed the real-time PCR analysis and analyzed the results. BJK (Byoung-Jun Kim), BRK, SYL, and GNK prepared the genomic DNA used in this study. TSS provided the sputum samples. YHK interpreted the data and was involved in drafting the manuscript. BJK (Bum-Joon Kim) conceived the study, participated in the study design and drafted the manuscript. All authors read and approved the final manuscript.

\section{Acknowledgements}

This research was supported by a grant (Grant No., H113C15620000) from the Korean Heathcare Technology R\&D project, Ministry for Health, Welfare \& Family Affairs. Kijeong Kim and Seok-Hyun Hong equally contributed to this work.

\section{Author details}

${ }^{1}$ Department of Biomedical Sciences, Microbiology and Immunology, Cancer Research Institute, and Liver Research Institute, Seoul National University College of Medicine, 28 Yongon-dong, Chongno-gu, Seoul 110-799, Republic of Korea. ${ }^{2}$ Department of Microbiology, Chung-Ang University College of Medicine, Seoul 
156-756, Republic of Korea. ${ }^{3}$ Division of Pulmonary and Critical Care Medicine, Department of Internal Medicine, Asan Medical Center, University of Ulsan College of Medicine, Seoul, Republic of Korea.

\section{Received: 11 May 2015 Accepted: 31 July 2015}

\section{Published online: 11 August 2015}

\section{References}

1. Harris KA, Kenna DT, Blauwendraat C, Hartley JC, Turton JF, Aurora P, et al. Molecular fingerprinting of Mycobacterium abscessus strains in a cohort of pediatric cystic fibrosis patients. J Clin Microbiol. 2012;50(5):1758-61.

2. Maurer FP, Ruegger V, Ritter C, Bloemberg GV, Bottger EC. Acquisition of clarithromycin resistance mutations in the $23 \mathrm{~S}$ rRNA gene of Mycobacterium abscessus in the presence of inducible erm(41). J Antimicrob Chemoth 2012:67(11):2606-11.

3. Choi WS, Kim MJ, Park DW, Son SW, Yoon YK, Song T, et al. Clarithromycin and amikacin vs. clarithromycin and moxifloxacin for the treatment of post-acupuncture cutaneous infections due to Mycobacterium abscessus: a prospective observational study. Clin Microbiol Infect. 2011;17(7):1084-90.

4. Nash KA, Brown-Elliott BA, Wallace RJ. A novel gene, erm(41), confers inducible macrolide resistance to clinical isolates of Mycobacterium abscessus but is absent from Mycobacterium chelonae. Antimicrob Agents Chemother. 2009;53(4):1367-76

5. Kim HY, Kook Y, Yun YJ, Park CG, Lee NY, Shim TS, et al. Proportions of Mycobacterium massiliense and Mycobacterium bolletii strains among Korean Mycobacterium chelonae-Mycobacterium abscessus group isolates. J Clin Microbiol. 2008;46(10):3384-90.

6. Leao SC, Tortoli E, Viana-Niero C, Ueki SYM, Lima KVB, Lopes ML, et al. Characterization of mycobacteria from a Major Brazilian outbreak suggests that revision of the taxonomic status of members of the Mycobacterium chelonae-M-abscessus group is needed. J Clin Microbiol. 2009;47(9):2691-8.

7. Macheras E, Roux AL, Bastian S, Leao SC, Palaci M, Sivadon-Tardy V, et al. Multilocus sequence analysis and $r p o B$ sequencing of Mycobacterium abscessus (Sensu Lato) strains. J Clin Microbiol. 2011;49(2):491-9.

8. Leao SC, Tortoli E, Euzeby JP, Garcia MJ. Proposal that Mycobacterium massiliense and Mycobacterium bolletii be united and reclassified as Mycobacterium abscessus subsp bolletii comb. nov., designation of Mycobacterium abscessus subsp abscessus subsp nov and emended description of Mycobacterium abscessus. Int J Syst Evol Microbiol. 2011;61:2311-3.

9. Kim BJ, Yi SY, Shim TS, Do SY, Yu HK, Park YG, et al. Discovery of a Nove hsp65 Genotype within Mycobacterium massiliense associated with the rough colony morphology. Plos One. 2012;7(6):e38420.

10. Kim BJ, Kim BR, Hong SH, Seok SH, Kook YH, Kim BJ. Complete Genome Sequence of Mycobacterium massiliense Clinical Strain Asan 50594, Belonging to the Type II Genotype. Genome Announc. 2013;1(4) pii: e00429-13. doi: 10.1128/genomeA.00429-13.

11. Kim BJ, Kim BR, Lee SY, Kook YH, Kim BJ. Rough colony morphology of Mycobacterium massiliense Type II genotype is due to the deletion of glycopeptidolipid locus within its genome. BMC Genomics. 2013;14:890.

12. Jeon SM, Lim NR, Kwon SJ, Shim TS, Park MS, Kim BJ, et al. Analysis of species and intra-species associations between the Mycobacterium abscessus complex strains using pulsed-field gel electrophoresis (PFGE) and multi-locus sequence typing (MLST). J Microbiol Methods. 2014;104:19-25.

13. Leao SC, Viana-Niero C, Matsumoto CK, Lima KV, Lopes ML, Palaci M, et al. Epidemic of surgical-site infections by a single clone of rapidly growing mycobacteria in Brazil. Future Microbiol. 2010;5(6):971-80.

14. Aitken ML, Limaye A, Pottinger P, Whimbey E, Goss CH, Tonelli MR, et al. Respiratory outbreak of Mycobacterium abscessus subspecies massiliense in a lung transplant and cystic fibrosis center. Am J Respir Crit Care Med. 2012;185(2):231-2.

15. Bryant JM, Grogono DM, Greaves D, Foweraker J, Roddick I, Inns T, et al. Whole-genome sequencing to identify transmission of Mycobacterium abscessus between patients with cystic fibrosis: a retrospective cohort study. Lancet. 2013;381(9877):1551-60.

16. Bastian S, Veziris N, Roux AL, Brossier F, Gaillard JL, Jarlier V, et al. Assessment of Clarithromycin Susceptibility in Strains Belonging to the Mycobacterium abscessus Group by erm(41) and rrl Sequencing. Antimicrob Agents Chemother. 2011;55(2):775-81.

17. Shallom SJ, Gardina PJ, Myers TG, Sebastian Y, Conville P, Calhoun LB, et al. New rapid scheme for distinguishing the subspecies of the Mycobacterium abscessus group and identifying Mycobacterium massiliense isolates with inducible clarithromycin resistance. J Clin Microbiol. 2013;51(9):2943-9.

18. Porcheddu A, Giacomelli G. Peptide nucleic acids (PNAs), a chemical overview. Curr Med Chem. 2005;12(22):2561-99.

19. Choi YJ, Kim HS, Lee SH, Park JS, Nam HS, Kim HJ, et al. Evaluation of peptide nucleic acid array for the detection of hepatitis $B$ virus mutations associated with antiviral resistance. Archives of virology. 2011;156(9):1517-24.

20. Kim JW, Choi YJ, Kim HJ, Park JS, Nam HS, Hwangbo Y, et al. Comparison of PNA probe-based real-time PCR and Cobas TaqMan MTB for detection of MTBC. Biochip J. 2013:7(2):85-8.

21. Choi YJ, Kim HJ, Shin HB, Nam HS, Lee SH, Park JS, et al. Evaluation of peptide nucleic acid probe-based real-time PCR for detection of Mycobacterium tuberculosis complex and nontuberculous mycobacteria in respiratory specimens. Ann Lab Med. 2012;32(4):257-63.

22. Kim K, Kim BJ, Shim TS, Hong SH, Kook YH, Kim BJ. Development of a Peptide nucleic acid-based multiprobe real-time PCR method targeting the hsp65 gene for differentiation among Mycobacterium abscessus strains. J Clin Microbiol. 2015;53(4):1403-5.

23. Kim K, Lee H, Lee MK, Lee SA, Shim TS, Lim SY, et al. Development and application of multiprobe real-time PCR method targeting the hsp65 gene for differentiation of Mycobacterium species from isolates and sputum specimens. J Clin Microbiol. 2010;48(9):3073-80.

24. Kent PK,G. Public Health Mycobacteriology. A Guide for the Level III Laboratory. Atlanta, GA: Centers for Disease Control and Prevention; 1985.

25. Taylor Z, Nolan CM, Blumberg HM, American Thoracic S, Centers for Disease C, Prevention, Infectious Diseases Society of A. Controlling tuberculosis in the United States. Recommendations from the American Thoracic Society, CDC, and the Infectious Diseases Society of America. MMWR Recomm Rep. 2005;54(RR-12):1-81

26. Kim BJ, Lee SH, Lyu MA, Kim SJ, Bai GH, Chae GT, et al. Identification of mycobacterial species by comparative sequence analysis of the RNA polymerase gene (rpoB). J Clin Microbiol. 1999;37(6):1714-20.

27. Schaaf CP, Wiszniewska J, Beaudet AL. Copy number and SNP arrays in clinical diagnostics. Annu Rev Genom Hum G. 2011;12:25-51.

28. Tang JF, Zhou L, Liu XY, Zhang CM, Zhao YY, Wang YF. Novel multiplex real-time PCR system using the SNP technology for the simultaneous diagnosis of Chlamydia trachomatis, Ureaplasma parvum and Ureaplasma urealyticum and genetic typing of serovars of $C$. trachomatis and $U$. parvum in NGU. Mol Cell Probe. 2011;25(1):55-9.

29. Eeles RA, Kote-Jarai Z, Giles GG, Olama AA, Guy M, Jugurnauth SK, et al. Multiple newly identified loci associated with prostate cancer susceptibility. Nat Genet. 2008:40(3):316-21.

30. Goel G, Kumar A, Puniya AK, Chen W, Singh K. Molecular beacon: a multitask probe. J Appl Microbiol. 2005;99(3):435-42.

31. Zhang N, Appella DH. Colorimetric detection of anthrax DNA with a Peptide nucleic acid sandwich-hybridization assay. J Am Chem Soc. 2007;129(27):8424-5.

32. Grossmann TN, Roglin L, Seitz O. Target-catalyzed transfer reactions for the amplified detection of RNA. Angew Chem Int Ed Engl. 2008;47(37):7119-22.

33. Petersen $K$, Vogel U, Rockenbauer E, Nielsen KV, Kolvraa S, Bolund L, et al. Short PNA molecular beacons for real-time PCR allelic discrimination of single nucleotide polymorphisms. Mol Cell Probes. 2004;18(2):117-22.

34. Nielsen P. Peptide Nucleic Acids-Protocol and Applications. Norfolk: Horizon Bioscience; 2004.

35. Funes-Huacca ME, Opel K, Thompson R, McCord BR. A comparison of the effects of PCR inhibition in quantitative PCR and forensic STR analysis. Electrophoresis. 2011;32(9):1084-9.

36. Opel KL, Chung D, McCord BR. A study of PCR inhibition mechanisms using real time PCR. J Forensic Sci. 2010;55(1):25-33. 